\title{
Influence of Curing Humidity on the Compressive Strength of Gypsum-Cemented Similar Materials
}

\author{
Weiming Guan,, Dongsheng Zhang, ${ }^{1,2}$ Yingyuan Wen, ${ }^{1,2}$ and Xufeng Wang \\ ${ }^{1}$ State Key Laboratory of Coal Resources and Safe Mining, China University of Mining and Technology, Xuzhou, Jiangsu 221008, China \\ ${ }^{2}$ College of Geological and Mining Engineering, Xinjiang University, Urumqi, Xinjiang 830000, China \\ Correspondence should be addressed to Dongsheng Zhang; dshzhang123@126.com
}

Received 19 July 2016; Revised 11 October 2016; Accepted 13 October 2016

Academic Editor: Hossein Moayedi

Copyright (c) 2016 Weiming Guan et al. This is an open access article distributed under the Creative Commons Attribution License, which permits unrestricted use, distribution, and reproduction in any medium, provided the original work is properly cited.

\begin{abstract}
The analogous simulation experiment is widely used in geotechnical and mining engineering. However, systematic errors derived from unified standard curing procedure have been underestimated to some extent. In this study, 140 gypsum-cemented similar material specimens were chosen to study their curing procedure with different relative humidity, which is $10 \%-15 \%, 40 \%, 60 \%$, and $80 \%$, respectively. SEM microstructures and XRD spectra were adopted to detect the correlation between microstructures and macroscopic mechanical strength during curing. Our results indicated that the needle-like phases of similar materials began to develop in the early stage of the hydration process through intersecting with each other and eventually transformed into matlike phases. Increase of humidity may inhibit the development of needle-like phases; thus the compressive strength changes more smoothly, and the time required for the material strength to reach the peak value will be prolonged. The peak strength decreases along with the increase of humidity while the humidity is higher than $40 \%$; however, the reverse tendency was observed if the humidity was lower than $40 \%$. Finally, we noticed that the material strength usually reaches the peak value when the water content continuously reduces and tends towards stability. Based on the above observation, a curing method determination model and experimental strength predication method for gypsum-cemented similar materials were proposed.
\end{abstract}

\section{Introduction}

The analogous simulation experiment is a widely used approach in geotechnical engineering, especially in the field of coal mining, including the overlying strata movement fracture characteristics [1, 2], coal and gas outburst [3], backfilling technologies [4], and surface subsidence [5] after coal mining. However, certain inherent limitations have been observed in this approach. For example, since there are many artificial influence factors during experiments, the mechanical properties of rock similar materials are always not stable, leading to less reproducibility for the analytic result. Figures 1 (a) and 1(b) show the analogous simulation experiments performed for mining Xinjiang ultra-thick seam in June 2015. Both simultaneous experiments using the same apparatus and protocol were conducted at Xinjiang University (Urumqi, western inland of China) and China University of Mining and Technology (Xuzhou, eastern coast of China). However, the results were completely different. More specifically, in the experiment conducted at Xuzhou, fissures run through the surface and the main key strata fractured. In contrast, another experiment conducted at Urumqi, the main key strata did not fracture. Many factors may contribute to such variation, including the sand diameter, compaction degree, curing temperature, and curing humidity. To increase the accuracy and repeatability of the analogous simulation experiments, investigations on these factors are required in order to explore the experimental regularity and develop more reproducible experimental protocol.

At present, many studies regarding the analogous simulation materials mainly focus on the rock similar material mixing ratio and the experimental error analysis. Traditionally, the similar materials can be divided into three typical groups by the majority of cementation (i.e., gypsum, portland cement, and iron-crystal sand) [6-8]. Some researchers also developed novel rock similar materials to meet special demands, including transfusion and rock burst [9]. The mass mixing ratio also has a significant influence on the strengths 


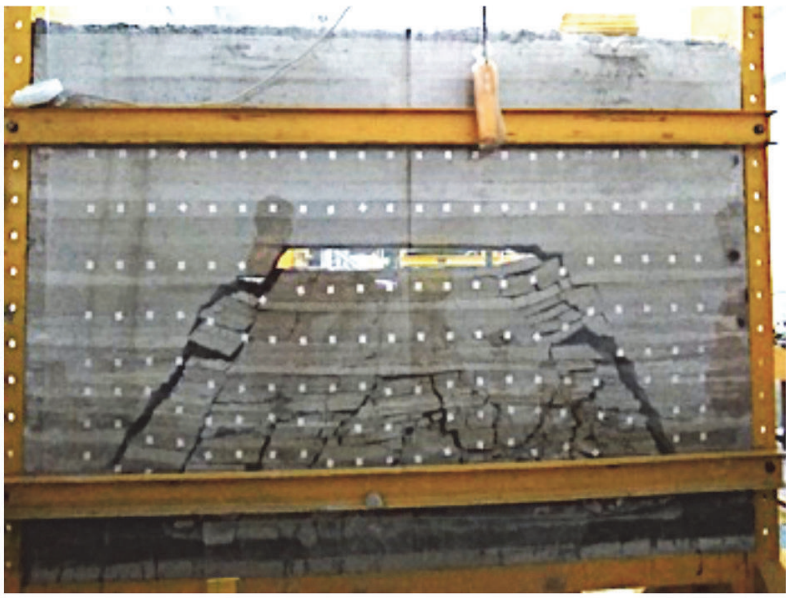

(a)

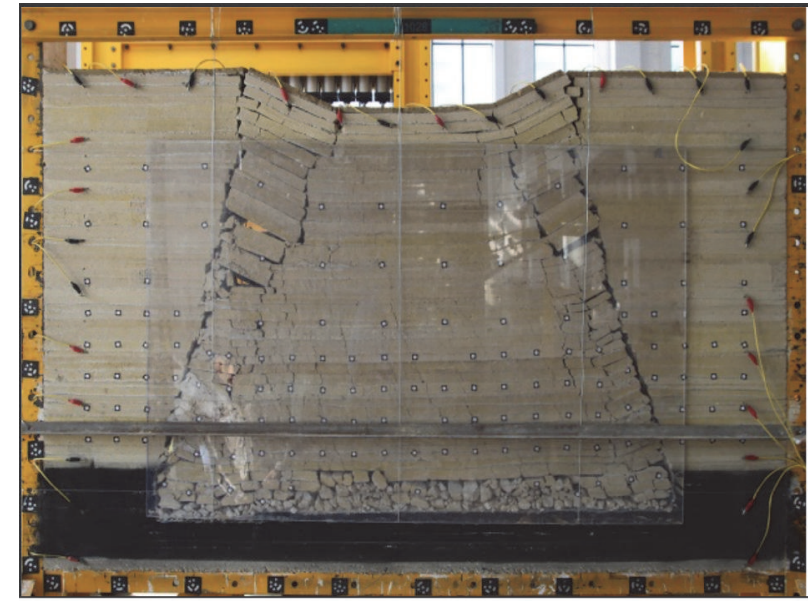

(b)

FIGURE 1: Results of analogous experiments conducted in different climate. Experiments conducted in Urumqi (a) and Xuzhou (b) in June.

as described by Chen et al. [10] and Kang et al. [11]. With regard to the resource of error in similar simulation experiments, Ximin et al. [12] classified them into three categories, including controllable, changeable, and unchangeable. Luo et al. [13] further analyzed the error source of analogous experiments during molding, curing, and testing based on experiments. Zhang et al. [14] systematically discussed the influence of shaping pressure on the similar material strength. Li et al. [15] and Jia et al. [16] explore the effects of different particle sizes of sand on mechanical properties of the similar material. Yuan and $\mathrm{Xu}$ [17] investigated the influence regularity of curing duration on the kinetic property of analogous materials based on Hopkinson tests.

However, there are few researchers who have noticed the relation between curing humidity and the strengths of similar materials. The mechanical strengths of similar materials are closely related to the hydration degree of cementing agents, and the curing humidity has a direct influence on the hydration reaction. The climate in Urumqi, Xinjiang, is a temperate continental arid one, whereas that in Xuzhou, Jiangsu, is a warm temperature semihumid monsoon climate. As displayed in Figure 2, obvious deviations exist between the monthly average relative humidity of the two cities. In Urumqi, the relative humidity in June is $45 \%$, which is $65 \%$ in the latter city. Thus, investigating the relationship between curing humidity and similar material mechanical strength is crucial to the improvement of accuracy for this type of experiment.

In this study, the variation regularity of the uniaxial compressive strength of a gypsum-cemented similar material under different curing humidity conditions was investigated with the same mixing scheme. Scanning Electron Microscopy (SEM), Energy Dispersive Spectrometer (EDS), and XRay Diffraction (XRD) analysis were applied to reveal the microstructure evolution and product generation process of the gypsum-cemented similar material. Finally, we proposed the mechanism of the macroscopic mechanical strength variation based on observed result and further constructed an

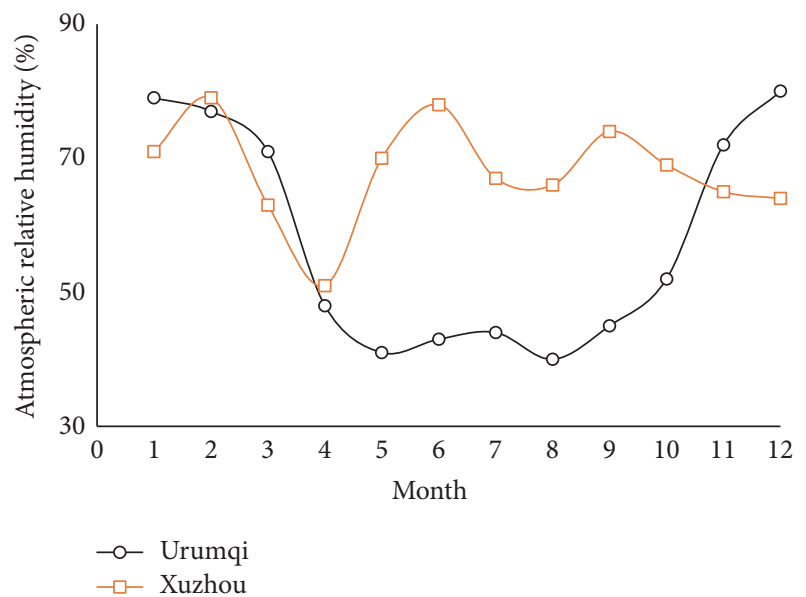

FIGURE 2: Monthly average relative humidity in Urumqi and Xuzhou $[18,19]$.

analogous experimental curing method determination model and material strength prediction method.

\section{Experimental Detail}

2.1. Material Mixing Scheme. The similar material used in this study was prepared by mixing $\beta$ semihydrate gypsum $\left(\mathrm{CaSO}_{4} \cdot 0.5 \mathrm{H}_{2} \mathrm{O}\right)$, calcium carbonate powder $\left(\mathrm{CaCO}_{3}\right)$, and washed-out sand which were stirred with water. As shown in Table 1, the virgin rock strength simulated was $34.6 \mathrm{MPa}$. According to the similarity criterion [20] and experimental condition, the model geometric proportion was decided as $1 / 230$, and the unit weight was selected as $1 / 1.5$. Thus, the material similar compressive strength was 34/6/230/1.5 = $100.2 \mathrm{kPa}$. As shown in Figure 3, the mixing ratio was 773. In the mixing ratio, the first 7 indicate that the mass percentage of sand is $7 / 8$, whereas the second 7 and the last 3 indicate that, in the remaining $1 / 8$ of mass, the $\mathrm{CaSO}_{4} \cdot 0.5 \mathrm{H}_{2} \mathrm{O}$ accounts for 
TABLE 1: Material mixing scheme and the size of raw material.

\begin{tabular}{|c|c|c|c|c|c|c|c|c|c|}
\hline Rock type & $\begin{array}{l}\text { Density } \\
\mathrm{g} / \mathrm{cm}^{3}\end{array}$ & $\begin{array}{l}\text { Compressive } \\
\text { strength/MPa }\end{array}$ & $\begin{array}{c}\text { Model } \\
\text { density } \\
\mathrm{g} / \mathrm{cm}^{3}\end{array}$ & $\begin{array}{c}\text { Model } \\
\text { compressive } \\
\text { strength } / \mathrm{kPa}\end{array}$ & $\begin{array}{l}\text { Mixing } \\
\text { ratio }\end{array}$ & $\begin{array}{l}\text { Water } \\
\text { percent }\end{array}$ & $\begin{array}{l}\text { Semihydrate } \\
\text { gypsum/mesh }\end{array}$ & $\begin{array}{c}\text { Calcium } \\
\text { carbonate } \\
\text { powder/mesh }\end{array}$ & $\begin{array}{l}\text { Washed-out } \\
\text { sand/mesh }\end{array}$ \\
\hline Mudstone & 1.7 & 34.6 & 1.13 & 100.2 & 773 & $1 / 9$ & $300-400$ & $300-400$ & $80-100$ \\
\hline
\end{tabular}

TABLE 2: Experiment scheme for analyzing the influence of curing humidity on similar material strength.

\begin{tabular}{lcccc}
\hline Time & $\begin{array}{c}\text { Indoor } \\
\text { environment } \\
(10 \%-15 \%)\end{array}$ & \multicolumn{3}{c}{$\begin{array}{c}\text { Conidity } \\
\text { Cumidity chamber }\end{array}$} \\
\hline $1 \mathrm{~d}$ & 5 & $50 \%$ & $60 \%$ & $80 \%$ \\
$2 \mathrm{~d}$ & 5 & 5 & 5 & 5 \\
$3 \mathrm{~d}$ & 5 & 5 & 5 & 5 \\
$4 \mathrm{~d}$ & 5 & 5 & 5 & 5 \\
$5 \mathrm{~d}$ & 5 & 5 & 5 & 5 \\
$7 \mathrm{~d}$ & 5 & 5 & 5 & 5 \\
$10 \mathrm{~d}$ & 5 & 5 & 5 & 5 \\
\hline
\end{tabular}

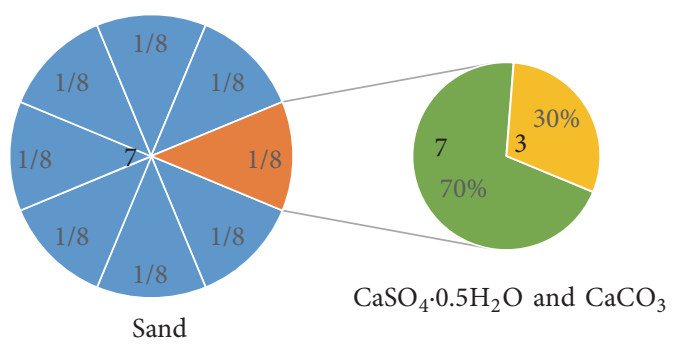

Figure 3: Mixing ratio: 773.

$70 \%$ and the $\mathrm{CaCO}_{3}$ for $30 \%$. Besides the water mass was $1 / 9$ of the total mass.

2.2. Experiment Scheme and Process. The relative humidity through the year in Urumqi varies between $40 \%$ and $80 \%$; average value of humidity is $55 \%$. A specific experiment scheme was designed (Table 2) to explore the influence of curing humidity on the similar material strength. Based on curing conditions, experiments were divided into four groups. Group 1 was maintained in a natural indoor environment, with a humidity of $10 \%-15 \%$ and a temperature of $25-$

$28^{\circ} \mathrm{C}$. Groups $2-4$ were maintained in constant temperature humidity chambers, with humidity values of $40 \%, 60 \%$, and $80 \%$. The temperature of Groups $2-4$ kept stable at $26^{\circ} \mathrm{C}$. Each group contained 35 specimens, and there were 140 specimens in total. To reduce artificial errors possibility, all specimens were prepared by the same staff on the same day and were placed into the curing environment for maintenance. Furthermore, it is also necessary to ensure consistent sample density. Thus, $5 \mathrm{~cm} * 10 \mathrm{~cm}$ PVC pipes were used as mold, and in the filling process every sample was weighed by

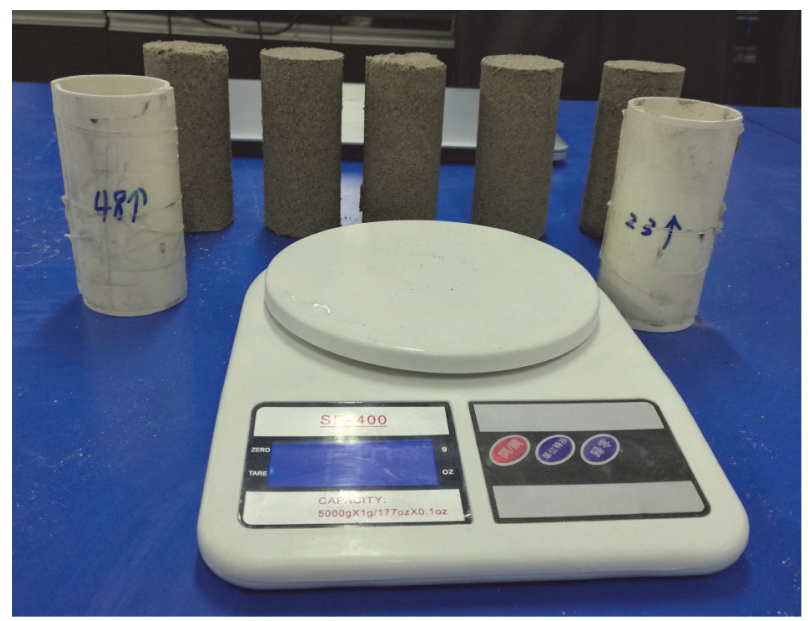

(a) Specimen mold and electronic scale

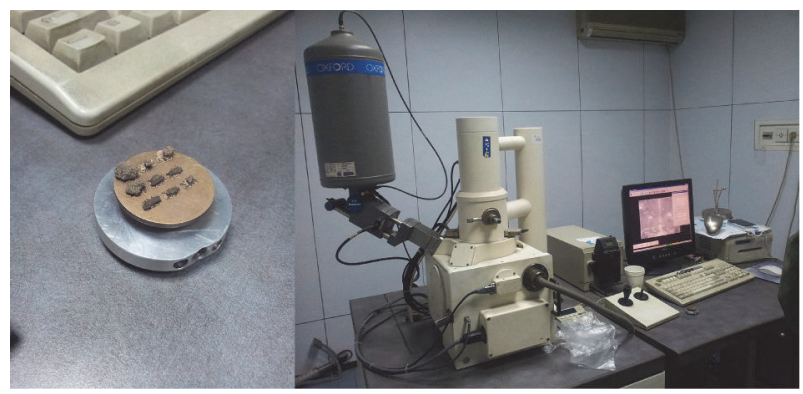

(b) SEM tests

FIGURE 4: Sample preparation tools and SEM tests.

electronic scale. The sample preparation tools are displayed in Figure 4(a).

To observe the strength variation of the similar material under different humidity conditions, five specimens were taken out from each group after curing for 1, 2, 3, 4, 5, 7, and 10 days. These specimens were uniaxially compressed, and the broken blocks were used for the water content measurement and SEM characterization. Figure 4(b) displays the SEM tests. Due to the comparably poor electrical conductivity of sand materials, metal spraying was needed before SEM observation.

\section{Results Analysis}

The strength generation of cemented similar materials is directly decided by the hydration reaction of the cementing materials. The hydration reaction primarily produces two-phase categories: new phases produced after gypsum 


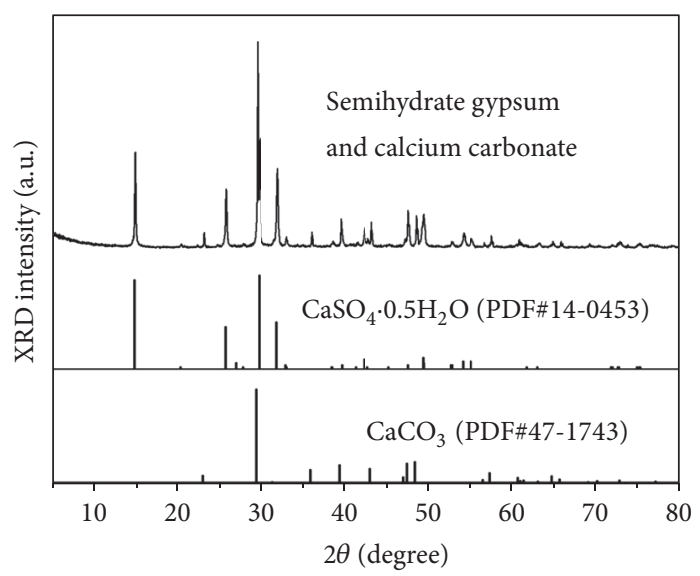

(a) Before reaction (mixture of calcium carbonate and semihydrate gypsum)

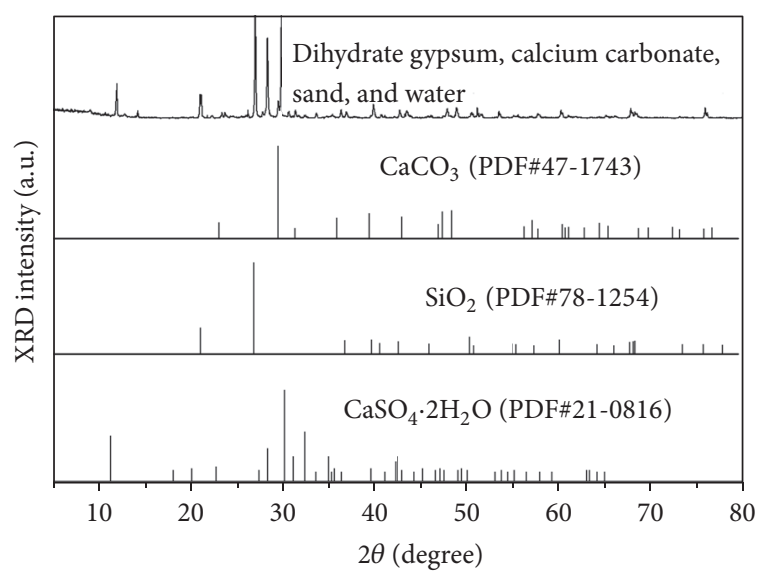

(b) After reaction (mixture of calcium carbonate, dihydrate gypsum, sand, and water)

FIGURE 5: XRD spectra of the gypsum-cemented similar material before and after hydrate reaction.

hydration and residual phases that are not or insufficiently involved in reactions. The new phases play the dominant role and are called the cementing phases. The type, amount, relative size, and spatial distribution of cementing phases determine the strength features of similar materials.

3.1. XRD and EDS Analysis of Hydration Products. To explore the composition of the gypsum hydration products, XRD was used to compare the phase compositions before and after hydration. Thus, the major reaction products were determined. During the SEM measurement, EDS was utilized to analyze the element contents in different areas of the crystallized product, and the chemical compositions of products with various morphologies were determined.

3.1.1. XRD Analysis. To determine the phase change after gypsum hydration, XRD characterization was conducted on the calcium carbonate and semihydrate gypsum mixture, and the similar material was maintained in the laboratory environment for 5 days. The diffraction spectra that were obtained are displayed in Figure 5 and were compared to the powder crystal database provided by ICDD (International Center for Diffraction Data). It was found that the composition before the hydration reaction consisted of $\mathrm{CaCO}_{3}$ and $\mathrm{CaSO}_{4} \cdot 0.5 \mathrm{H}_{2} \mathrm{O}$, whereas after the hydration reaction, it consisted of $\mathrm{CaCO}_{3}, \mathrm{CaSO}_{4} \cdot 2 \mathrm{H}_{2} \mathrm{O}$, and $\mathrm{SiO}_{2}$. Thus, it was determined that the cementing material used in this study is semihydrate gypsum, and the new phase formed during the reaction is dihydrate gypsum.

3.1.2. EDS Analysis. To determine the types of products with various morphologies, Energy Dispersive Spectrometer (EDS) analysis was conducted to characterize the type and amount of elements in the microregions. The regular sheetlike objects displayed in Figure 6 are typical semihydrate gypsum, while the needle-like objects are crystallized dihydrate gypsum, which is separated from the sheet-like ones.
3.2. Microstructure Characterization and Development Features. In order to understand the internal microstructure and related evolution process of gypsum-cemented similar material under various curing humidity and time conditions, SEM images were obtained according to the experimental scheme shown in Table 2, and these images can be found in Figure 7. The horizontal axis represents the humidity variation, that is, $10 \%-15 \%$ in a laboratory environment and $40 \%, 60 \%$, and $80 \%$ in constant temperature humidity chambers. The vertical axis stands for the time variation of $1 \mathrm{~d}, 2 \mathrm{~d}, 3 \mathrm{~d}, 4 \mathrm{~d}$, and $5 \mathrm{~d}$.

As displayed in Figure 7, when the curing duration is fixed, the amount of needle-like phases continuously reduces with increasing curing humidity, while the block and sheet phases increase. Under the same humidity condition, the intersecting degree of needle-like phases presents an increasing tendency as the curing duration is prolonged. However, the whole specimen begins to expand as the hydration reaction continues to proceed, and additional micropores occur.

3.3. Crystallization Process Analysis. Semihydrate gypsum $\left(\mathrm{CaSO}_{4} \cdot 0.5 \mathrm{H}_{2} \mathrm{O}\right)$ reacts with water and forms dihydrate gypsum $\left(\mathrm{CaSO}_{4} \cdot 2 \mathrm{H}_{2} \mathrm{O}\right)$. The saturated solution degree of semihydrate gypsum $\left(20^{\circ} \mathrm{C}, 8.85 \mathrm{~g} / \mathrm{L}\right)$ is much higher than that of the dihydrate gypsum $\left(20^{\circ} \mathrm{C}, 2.04 \mathrm{~g} / \mathrm{L}\right)$. Thus, the dihydrate gypsum solution is over saturated, and dihydrate crystals automatically form and grow up in the semihydrate gypsum solution. With the precipitation of dihydrate gypsum, the equilibrium concentration of the semihydrate gypsum reduced. At this point, the further dissolution of semihydrate gypsum increases the $\mathrm{CaSO}_{4}$ concentration. With the continuous dissolving of semihydrate gypsum, dihydrate gypsum continues crystallizing and growing until the $\mathrm{CaSO}_{4}$ concentration no longer increases, and the dihydrate gypsum cannot crystallize again.

The gypsum crystallization process can be divided into two stages: crystal nucleus production and crystal growth. Different relative supersaturation degrees decide the crystal nucleus distribution density and thus affect the morphology 


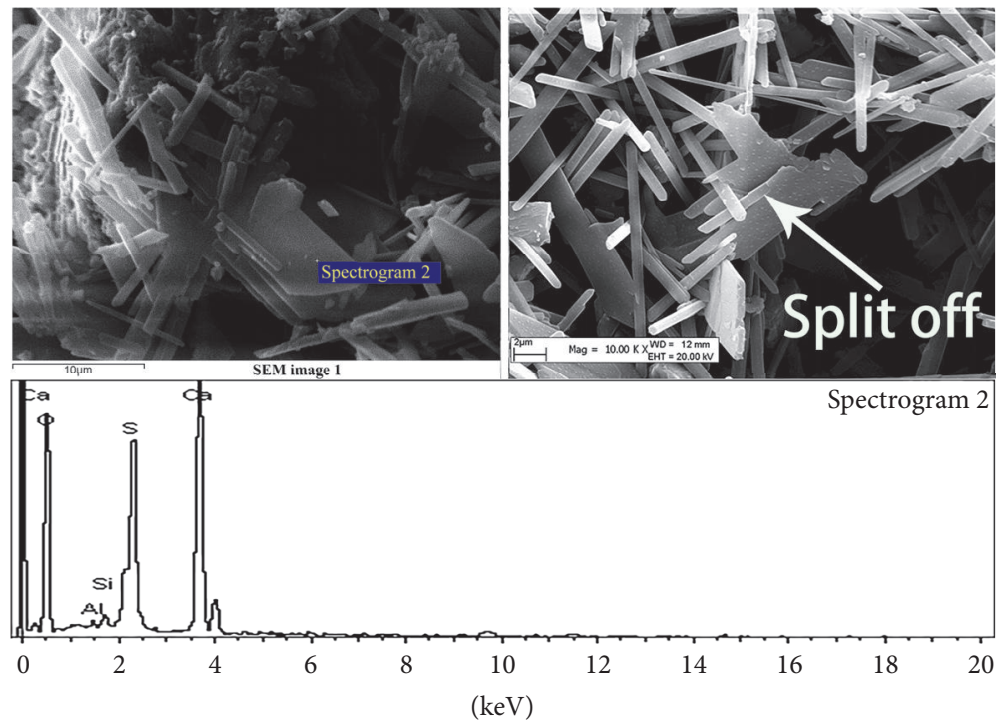

Full scale counts: $701 \mathrm{cts}$

FIGURE 6: Surface morphologies and chemical compositions before and after hydration reaction.

of the crystals growing to adhere to these nucleuses. If the supersaturation degree is relatively low, the nucleus distribution density is relatively small. Crystals grow up by adhering to nucleuses, and the volume of single crystals that are finally formed is large, most of which exist in the form of chunky bars. When the relative supersaturation degree increases, the production rate of nucleuses is accelerated, and the nucleus distribution density sharply rises. The volume of formed single crystals is small with needle-like morphologies. These needle-like phases intersect and form felt-like morphologies. The crystal growth gradually fills the pores between the sand particles, thus giving the similar material its strength. Afterward, the crystallization products keep increasing. The volume of specimen continuously expands with the increase of internal pores, resulting in a reduction in whole strength.

The water content in gypsum-cemented similar materials is comparably high, while the supersaturation degree of dihydrate gypsum inside specimens is relatively low. With the water inside the specimen continuously moving outward to the relatively dry environment, the supersaturation degree of dihydrate gypsum rises, and the crystallization rate also increases. Therefore, the variation of curing humidity mainly affects the migration velocity of water inside the specimen and thereby influences the supersaturation degree and crystallization rate of dihydrate gypsum. The higher the curing humidity is, the longer the low supersaturation degree of gypsum inside the specimen remains. Thus, it is clear that the higher the curing humidity is, the less the needle-like phases there are, but the most the block-like phases that exist.

\section{Curing Method Selection Model of Gypsum- Cemented Similar Material}

The curing method contains the curing duration and conditions. The construction of a characteristic model of strength,
TABLE 3: The relationship between curing humidity, time, and strength (uniaxial compressive strength, $\mathrm{kPa}$ ).

\begin{tabular}{lcccc}
\hline \multirow{2}{*}{ Time/d } & \multicolumn{4}{c}{ Humidity/\% } \\
& $10 \%-15 \%$ & $40 \%$ & $60 \%$ & $80 \%$ \\
\hline $1 \mathrm{~d}$ & $\mathbf{1 4 7 . 8}_{\max }$ & 98.6 & 55.3 & 28.7 \\
$2 \mathrm{~d}$ & 142.7 & $\mathbf{2 0 4 . 3 _ { \operatorname { m a x } }}$ & 77.1 & 38.2 \\
$3 \mathrm{~d}$ & 118.5 & 169.0 & 133.1 & 52.5 \\
$4 \mathrm{~d}$ & 107.0 & 136.9 & $\mathbf{1 5 7 . 3 _ { \operatorname { m a x } }}$ & 54.1 \\
$5 \mathrm{~d}$ & 104.3 & 123.6 & 126.9 & $\mathbf{1 0 0 . 4}$ \\
$7 \mathrm{~d}$ & 102.1 & 115.2 & 91.8 & 81.0 \\
$10 \mathrm{~d}$ & 97.1 & 112.5 & 79.4 & 61.2 \\
\hline
\end{tabular}

time, and humidity can provide a reference for selecting a reasonable curing humidity as well as reducing the curing period when conducting analogous model experiments.

4.1. Variation Regularity of Curing Duration, Humidity, and Material Strength. According to the test plan of Table 2, the variation data of curing duration, humidity, and compressive strength is obtained via uniaxial compression, as shown in Table 3.

4.1.1. Variation Regularity of Curing Duration and Strength. Figure 8(a) shows the relationship between the curing duration and material strength. It is implied that, under various curing humidity conditions, the compressive strengths of the similar material present a tendency of first rapidly increasing, then decreasing, and finally becoming steady in the end, as time continues. Moreover, the higher the curing humidity is, the more stable the strength variation becomes. In the natural laboratory environment, the relative humidity is small (10\%-15\%). The strengthening process might be completed within one day. After one day, the strength begins to decline 


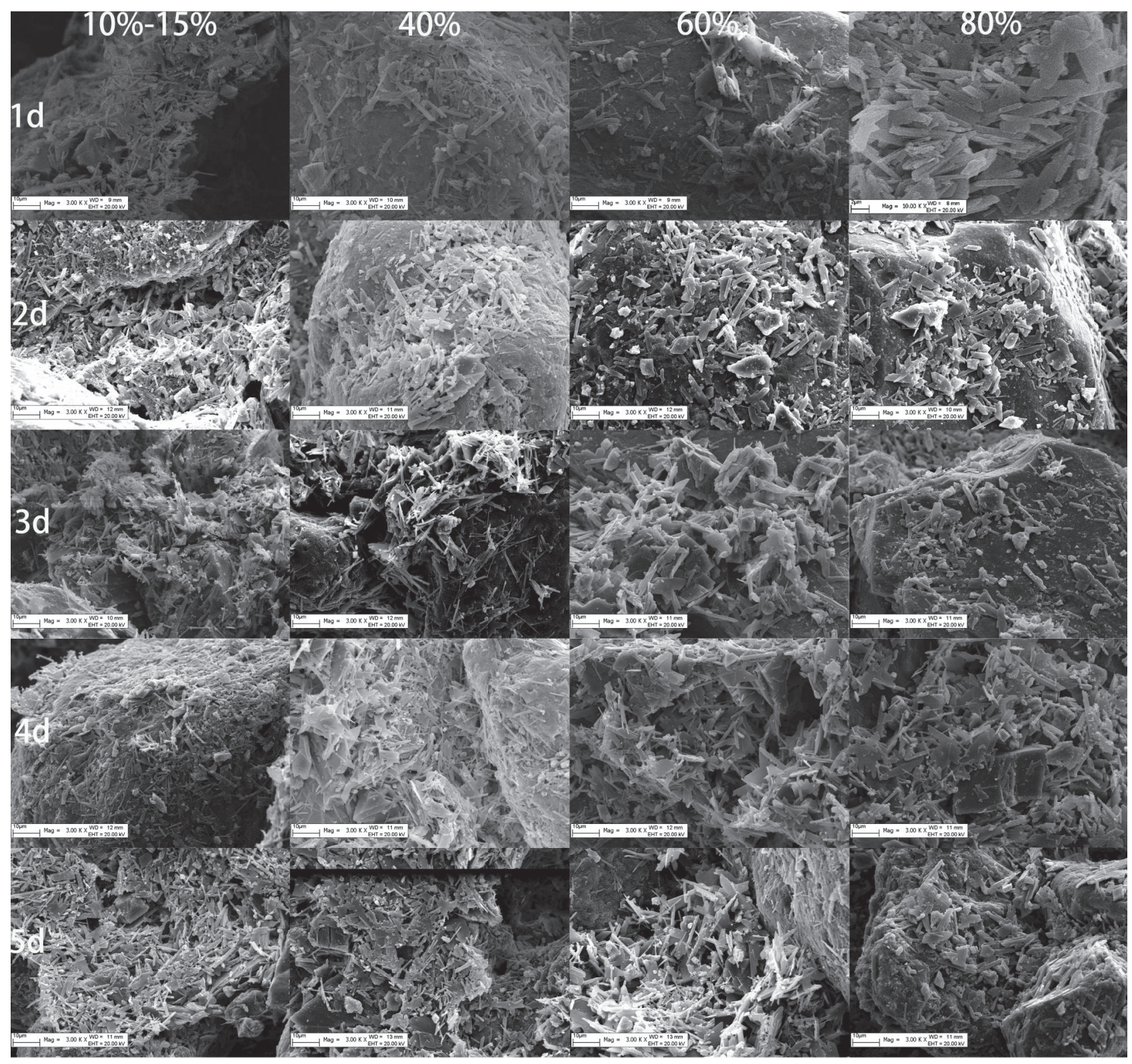

FIgURE 7: Microstructure characterization and development features of the gypsum-cemented similar material.

and reaches a stable value of $97.1 \mathrm{kPa}$ after ten days. When the relative humidity is $40 \%$, the material strength starts to decrease after two days and reaches a stable value of $112.5 \mathrm{kPa}$ after ten days. When the relative humidity is $60 \%$, the material strength begins to decrease after four days and reaches a stable value of $79.4 \mathrm{kPa}$ after ten days. When the relative humidity is $80 \%$, the strength reduction begins after five days and reaches $61.2 \mathrm{kPa}$ after ten days, still exhibiting an obvious decreasing tendency.

4.1.2. Variation Regularity of Curing Duration and Peak Material Strength. According to the peak strength curve displayed in Figure 8(b), the increase in curing humidity prolongs the time required to achieve material peak strength, that is, the first day, the second day, the fourth day, and the fifth day. The peak strength value slowly decreases along with the increase of humidity while the humidity is higher than $40 \%$; however, the reverse tendency was observed if the humidity was lower than $40 \%$.

4.1.3. Variation Regularity of Curing Humidity and Strength. Figure 8(c) displays the variation amplitude of material compressive strength under different curing conditions. From the figure, it can be seen that, with the increase in humidity, the overall strength initially increases and then decreases. The strength value range under the same humidity condition also presents a tendency of first increasing and then decreasing. In the laboratory environment, the material strength distributes 


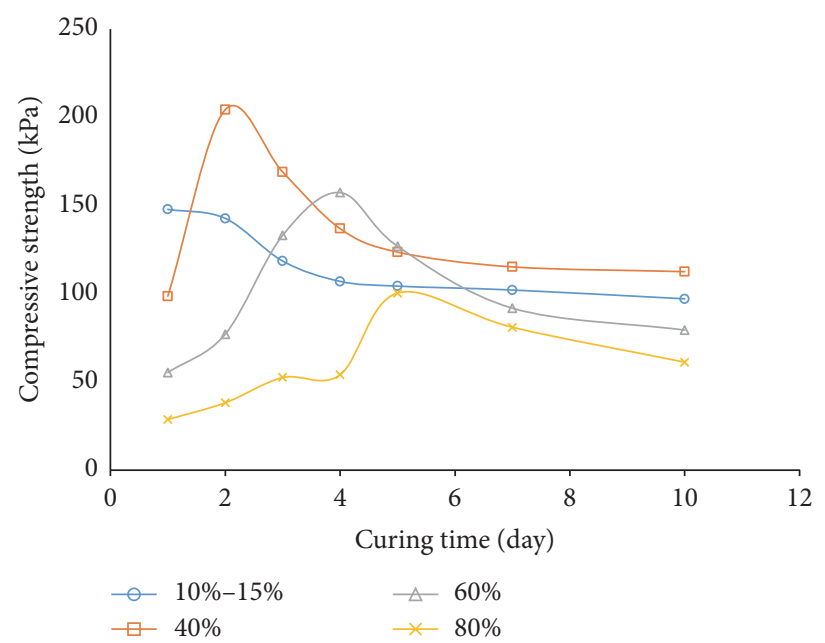

(a) Variation regularity of curing duration and material strength

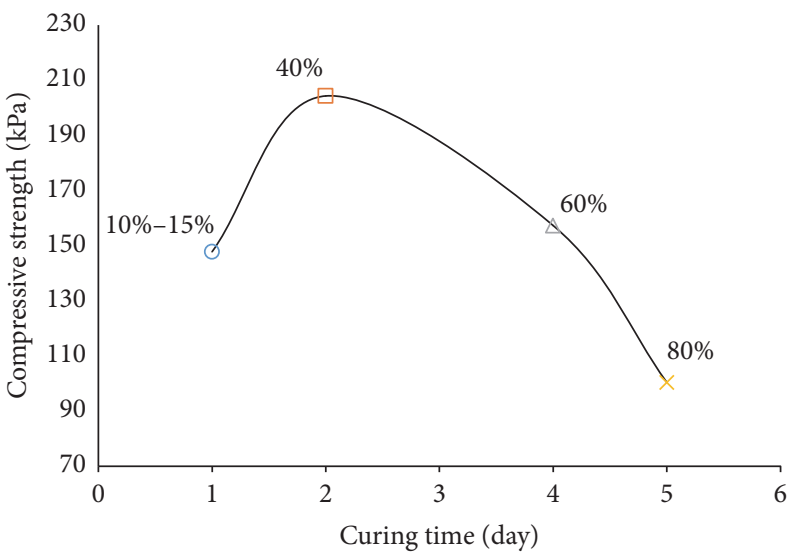

(b) Variation regularity of curing duration and material peak strength

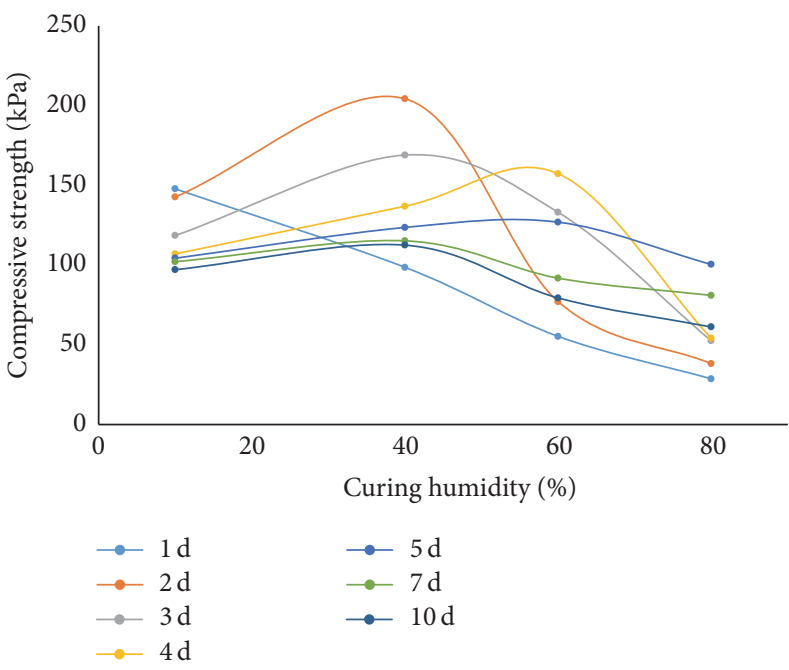

(c) Variation regularity of curing humidity and material strength

FIGURE 8: The relationship between curing duration, humidity, and strength.

most intensively $(97.1-147.8 \mathrm{kPa})$ with a range of $50.7 \mathrm{kPa}$. In contrast, in a humidity value of $40 \%$, the strength distributes most widely $(98.6-204.3 \mathrm{kPa})$ with a range of $105.7 \mathrm{kPa}$. The strength distribution range begins to shrink when the humidity is $60 \%(55.3-157.3 \mathrm{kPa})$, and the related range value is $103 \mathrm{kPa}$. For a humidity of $80 \%$, the variation strength range reduces further $(28.7-100.4 \mathrm{kPa})$, and the range value becomes $71.7 \mathrm{kPa}$.

\subsubsection{Mechanism Analysis of Curing Humidity Influencing} Material Strength. A certain degree of supersaturation and sufficient reaction time are required for the gypsum hydration. In high humidity environments (60\% and $80 \%)$, the water inside specimens slowly migrates outward, with a low relative supersaturation degree, low crystal nucleus distribution density, large crystal size, and large intercrystal pores. Thus, the material strength is reduced. Also, since the specimen matrix remains water-enriched for long periods, there is enough time for the dissolution and outward migration of various soluble substances, resulting in great changes in the specimen pore structures. Moreover, abundant water exists in the matrix pores and acts as a lubricant, thus decreasing the interparticle friction force and reducing the macroscopic strength. As displayed in Figure 9, the curing humidity increases from A to D. It can be seen that the different specimens have obviously different surface erosion conditions. For example, in comparing A and D, it is evident that Condition $\mathrm{D}$ has a greater number of erosion pores on the surface, which reduces the overall material strength.

Since the relative humidity in the laboratory environment is very low, the water inside the specimens moves quickly to the dry air, resulting in a rapid increase in gypsum supersaturation degree. The crystal nucleuses distribute intensively with small grain sizes. The intercrystal pores are small, and the material strength is strong. However, since the dry air continues to absorb water from the specimen, there is a lack of water for the hydration reaction, and the crystallization rate is reduced. Thus, in environments with extremely low 


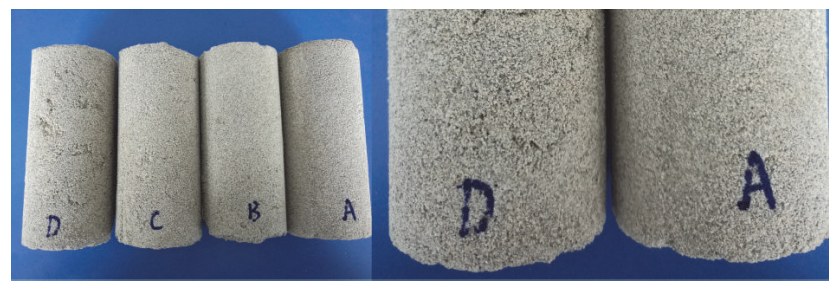

Figure 9: Surface erosion conditions of specimens under various curing humidity conditions.

humidity, low strength values occur after the specimen strength becomes stable.

The curing humidity affects the supersaturation degree of gypsum solution. Both overdry (10\%) and overwet (80\%) curing environments will lead to a reduction of similar material strength, but the strength variation amplitude tends to be stable. Moderate humidity (40\%) is beneficial for improving material strength, but the material strength experiences serious changes. Thus, during curing, the humidity should be carefully controlled according to the strength requirement and practical humidity conditions.

4.2. Selection Model of Similar Material Curing Humidity. The material strength peak point and the earliest strength stabilization inflection point are key points of material strength variation. According to the variation of material strength with curing duration, it can be divided into three zones: the strength enhancing zone (before peak value), strength reducing zone (between the peak value and stabilization inflection point), and strength stable zone (after stabilization inflection point).

The strength stable zone attracts the most attention during experiments. The time required to reach the earliest stable strength under various conditions and the stabilized strength average value are important data for improving experiment accuracy and efficiency. The peak point information under various curing conditions can be obtained from Figure 8(b). To acquire information about the stabilization inflection points, data in the reducing zone and the stable zone is fitted, with the obtained long-term strength variation curve and fitted equation being as follows:

$$
\begin{aligned}
& 38.19+\frac{1176.24}{x}+\frac{-8114.64}{x^{2}}+\frac{25749.1}{x^{3}}-\frac{34853.61}{x^{4}} \\
& +\frac{16152.52}{x^{5}} \\
& 104.98+\frac{184.57}{x}-\frac{1968.05}{x^{2}}+\frac{9929.89}{x^{3}}-\frac{11875.06}{x^{4}} ; \\
& 60.85+\frac{31.05}{x}+\frac{1433.36}{x^{2}} \\
& 23.42+\frac{389.16}{x}
\end{aligned}
$$

TABLE 4: The relationship between the strength increase rate and curing humidity (uniaxial compressive strength variation rate, $\mathrm{kPa} / \mathrm{d})$.

\begin{tabular}{lcccc}
\hline \multirow{2}{*}{ Time periods } & \multicolumn{4}{c}{ Humidity } \\
& $10 \%-15 \%$ & $40 \%$ & $60 \%$ & $80 \%$ \\
\hline $1 \mathrm{~d}-2 \mathrm{~d}$ & -5.10 & 105.70 & 21.80 & 9.50 \\
$2 \mathrm{~d}-3 \mathrm{~d}$ & -24.21 & -35.30 & 56.00 & 14.30 \\
$3 \mathrm{~d}-4 \mathrm{~d}$ & -11.45 & -32.11 & 24.20 & 1.60 \\
$4 \mathrm{~d}-5 \mathrm{~d}$ & -2.79 & -13.28 & -33.80 & 46.30 \\
$5 \mathrm{~d}-7 \mathrm{~d}$ & $-\mathbf{1 . 0 6}$ & -4.21 & -14.93 & -11.12 \\
$7 \mathrm{~d}-10 \mathrm{~d}$ & -1.68 & $-\mathbf{0 . 9 0}$ & -5.42 & -5.56 \\
$10 \mathrm{~d}-11 \mathrm{~d}$ & -1.97 & -0.36 & -2.77 & -3.54 \\
$11 \mathrm{~d}-12 \mathrm{~d}$ & -1.98 & -0.28 & -2.13 & -2.95 \\
$12 \mathrm{~d}-13 \mathrm{~d}$ & -1.95 & -0.23 & -1.67 & -2.49 \\
$13 \mathrm{~d}-14 \mathrm{~d}$ & -1.89 & -0.20 & -1.34 & -2.14 \\
$14 \mathrm{~d}-15 \mathrm{~d}$ & -1.81 & -0.19 & $-\mathbf{1 . 0 9}$ & -1.85 \\
$15 \mathrm{~d}-16 \mathrm{~d}$ & -1.73 & -0.17 & -0.90 & -1.62 \\
$16 \mathrm{~d}-17 \mathrm{~d}$ & -1.64 & -0.16 & -0.75 & -1.43 \\
$17 \mathrm{~d}-18 \mathrm{~d}$ & -1.55 & -0.16 & -0.64 & $-\mathbf{1 . 2 7}$ \\
$18 \mathrm{~d}-19 \mathrm{~d}$ & -1.47 & -0.15 & -0.54 & -1.14 \\
$19 \mathrm{~d}-20 \mathrm{~d}$ & -1.39 & -0.14 & -0.47 & -1.02 \\
\hline
\end{tabular}

TABLE 5: Strength, earliest stabilization time, and curing humidity in the stable zone.

\begin{tabular}{lccc}
\hline $\begin{array}{l}\text { Stable average } \\
\text { strength/kPa }\end{array}$ & Humidity/\% & $\begin{array}{c}\text { Earliest } \\
\text { stabilization } \\
\text { time/d }\end{array}$ & $\begin{array}{c}\text { Peak strength } \\
\text { time/d }\end{array}$ \\
\hline 90.23 & $10-15$ & 5 & 1 \\
111.65 & 40 & 7 & 2 \\
67.87 & 60 & 14 & 4 \\
43.94 & 80 & 17 & 5 \\
\hline
\end{tabular}

$$
\begin{aligned}
& -54.87+1801.66 * \frac{\ln (x)}{x}-5941478.43 * \exp (-x) \\
& 4.2+0.0009 * x^{2.5}-0.00007 * x^{3}
\end{aligned}
$$

Based on (1)-(4), the strength variation data within 20 days under various humidity conditions can be calculated as well as the material strength variation rates shown in Table 4 . The positive value indicates increased strength, while the negative value indicates reduced strength. The greater the absolute value is, the more obvious the strength change during this period is. Otherwise, the strength variation becomes stable.

The earliest strength stabilization inflection point should occur after the strength variation gradient begins to continuously reduce and tends towards stability. According to Figure 10(a), it is implied that the moment at which the absolute value of the gradient reaches 1 for the first time is the earliest strength stabilization inflection point. The average strength after stabilization can be decided by averaging all the strength values after the stabilization inflection point. The stabilization inflection points under various humidity conditions are shown in Table 5. 


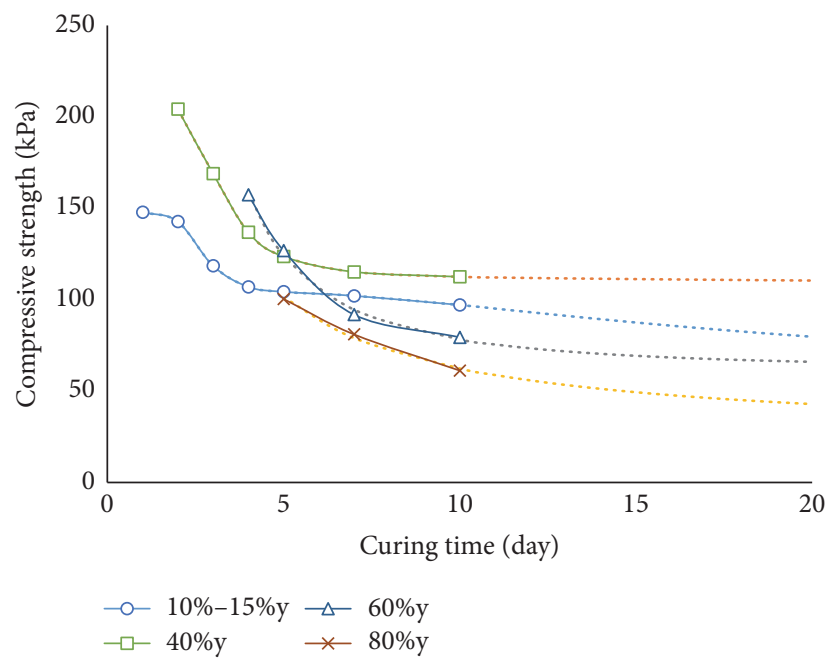

(a) Variation regularity and fitting function of strength in the reduction zone and the stable zone

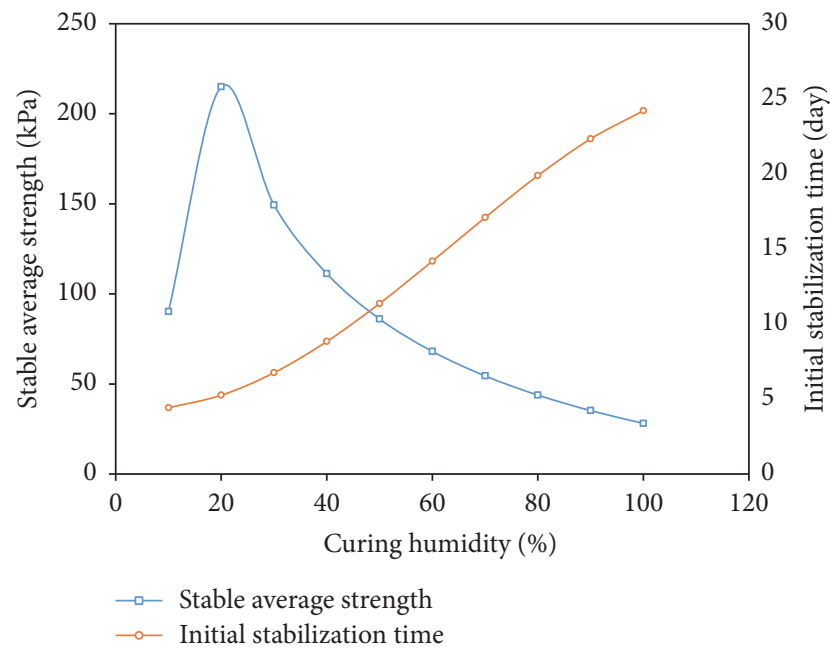

(b) The relationship between the initial stabilization time, stable average strength, and curing humidity

FIGURE 10: Selection model of the curing method of similar materials.

To construct the selection model for curing method, the relationship curve of humidity, strength, and time can be plotted, as displayed in Figure 10(b). It can be seen that when the curing humidity is greater than $40 \%$, the strength reduces very quickly after stabilization, and the time required for strength stabilization extends greatly. When the curing humidity is less than $40 \%$, the stable strength increases with curing humidity, and the stabilization time changes little, remaining within 7 days.

Equations (5) and (6) are fitted functions. According to the analogous simulation strength required in experiments, reasonable curing humidity and related earliest strength stabilization time can be calculated to improve the accuracy and efficiency of experiments.

The analogous specific strength in this study is $100.2 \mathrm{kPa}$. By substituting it into (5), the two curing humidity values can be solved as $10.04 \%$ and $43.95 \%$, which correspond to stabilization times of 4.41 and 9.78 days. Related curing conditions can be decided according to the humidity controllability and experimental efficiency.

\section{Material Strength Prediction Method in Analogous Experiments}

The variation of water content is an index of the water environment change during gypsum hydration. During analogous simulation experiments, the strength variation stage of similar material can be decided by continuously observing the water content.

5.1. Variation Regularity of Water Content and Time. Table 6 shows the variation of water contents under different curing humidity conditions. Figure 11 is the variation curve of water content with curing duration. Under all the conditions, the water content first rapidly decreases, and then, the fluctuation
TABLE 6: Variation relationship of curing humidity, time, and water content (the water content is in mass percentage).

\begin{tabular}{lcccc}
\hline Time & \multicolumn{5}{c}{ Humidity } \\
& $10 \%-15 \%$ & $40 \%$ & $60 \%$ & $80 \%$ \\
\hline $1 \mathrm{~d}$ & 2.95 & 5.67 & 6.72 & 7.88 \\
$2 \mathrm{~d}$ & 0.58 & 0.98 & 4.32 & 6.88 \\
$3 \mathrm{~d}$ & 0.82 & 1.46 & 2.88 & 6.65 \\
$4 \mathrm{~d}$ & 1.54 & 1.19 & 1.01 & 4.94 \\
$5 \mathrm{~d}$ & 0.78 & 1.17 & 1.37 & 3.43 \\
$6 \mathrm{~d}$ & 1.03 & 0.72 & 1.09 & 1.46 \\
$7 \mathrm{~d}$ & 0.74 & 0.86 & 0.89 & 1.06 \\
$10 \mathrm{~d}$ & 0.59 & 0.68 & 0.74 & 0.89 \\
\hline
\end{tabular}

tends towards stability. The greater the curing humidity is, the more stable the water content reduction is.

The evolution of water content in the similar material is made up of the gypsum crystallization water consumption and internal-external water exchange. The crystallization water consumption reduces the water partial pressure inside the specimens, making it possible for external water to diffuse inward and form humidity exchange. Thus, the water content is in a state of fluctuation. When the curing humidity is large, the humidity exchange is slow, and the crystallization water consumption is the major cause of water content reduction. When the curing humidity is small, the humidity exchange becomes rapid, which increases the supersaturation degree of gypsum inside the specimen and accelerates the crystallization rate. Under the combined action of crystallization and humidity exchange, the water content decreases very quickly.

5.2. Variation Regularity of Water Content and Strength. Figures $12(\mathrm{a})-12(\mathrm{~d})$ are curves illustrating the material strength and water content variation with time under various curing 


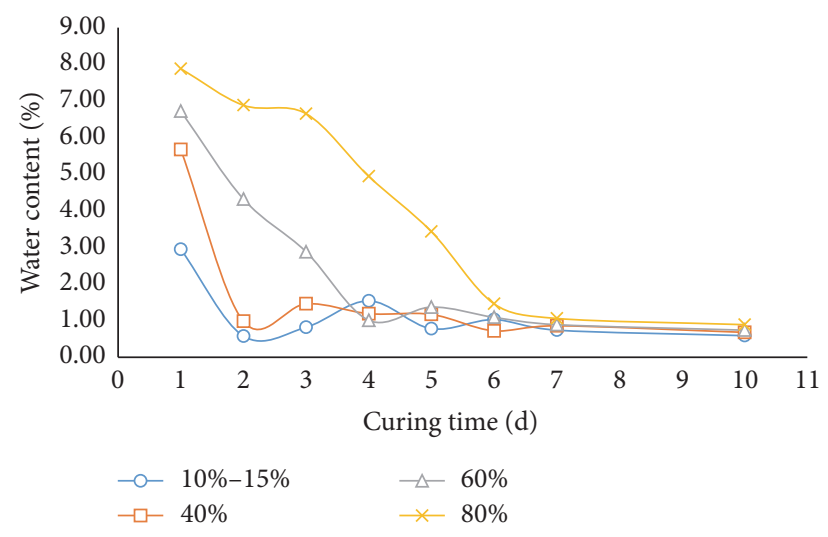

FIgURE 11: Variation of water content in the gypsum-cemented similar material.

TABLE 7: Water content variation rate (water content variation rate, $\% / d)$.

\begin{tabular}{lcccc}
\hline \multirow{2}{*}{ Time period } & \multicolumn{4}{c}{ Humidity } \\
& $10 \%-15 \%$ & $40 \%$ & $60 \%$ & $80 \%$ \\
\hline $1 \mathrm{~d}-2 \mathrm{~d}$ & -2.37 & -4.69 & -2.40 & -0.99 \\
$2 \mathrm{~d}-3 \mathrm{~d}$ & $\underline{\mathbf{0 . 2 4}}$ & $\underline{\mathbf{0 . 4 8}}$ & -1.43 & -0.23 \\
$3 \mathrm{~d}-4 \mathrm{~d}$ & 0.72 & -0.27 & -1.87 & -1.71 \\
$4 \mathrm{~d}-5 \mathrm{~d}$ & -0.76 & -0.03 & $\underline{\mathbf{0 . 3 6}}$ & -1.51 \\
$5 \mathrm{~d}-6 \mathrm{~d}$ & 0.24 & -0.45 & -0.27 & -1.97 \\
$6 \mathrm{~d}-7 \mathrm{~d}$ & -0.29 & 0.15 & -0.21 & $\underline{-\mathbf{0 . 4 0}}$ \\
$7 \mathrm{~d}-10 \mathrm{~d}$ & -0.05 & -0.06 & -0.05 & -0.06 \\
\hline
\end{tabular}

conditions. In the laboratory environment, the water content increases while the strength reduces. When the humidity is $40 \%-80 \%$, a negative correlation exists between the strength and water content before the peak strength; that is, the water content reduces while the strength increases.

Moreover, the water contents at all curing conditions quickly reduce to certain points, after which, the water content variation rate begins to become stable. At the moment, the material strength usually achieves its peak value. This is because the sample contains a lot of pores at the beginning of the reaction, and plenty of water is stored in there. As the crystal growth gradually fills the pores between the sand particles, thus giving the similar material its strength, the water is consumed and squeezed out. As a result, the water content continues to be reduced. When the pores are substantially filled, the material strength usually achieves its peak value, and the water content is reduced to its lowest value. However, the reaction does not stop and the crystallization products continue to increase. The specimen volume continuously expands with increasing internal pores, thus resulting in a reduction in whole strength. At this moment, there is a dynamic balance between the crystallization water consumption and the humidity exchange water supply.

Table 7 shows the water content variation condition. The positive value denotes an increase, whereas the negative value denotes a decrease. The lower the absolute value is, the more stable the water content variation becomes. When the water content continuously reduces, the variation rate is negative. When the water content becomes stable, the absolute rate is less than 1 . Thus, it can be determined that the time required to reach the peak strength under various conditions is $2 \mathrm{~d}$, $2 \mathrm{~d}, 4 \mathrm{~d}$, and $6 \mathrm{~d}$, respectively, which is different from the peak strength time displayed in Figure 8(b). This is attributed to the time interval setting during the strength test.

For example, under a humidity of $80 \%$, the tested peak strength occurs after 5 days; however, it is determined that the peak strength should occur on the sixth day according to the water content. This is because the time interval of the strength test is relatively large, and as a result, the strength data on the sixth day is not acquired. Thus, it is demonstrated that the variation in water content can accurately reflect the time required for the material to reach its peak strength.

\subsection{Prediction Method of Material Strength in Analogous} Simulation Experiments. First, the strength of the similar material should be decided according to experiment requirements. Then, the curing humidity and the time required to reach the stable strength are decided based on the curing method model ((5) and (6)). During the experiment, the variation condition of the water content is continuously monitored so as to characterize the moment at which the material strength reaches a peak value. By combining this with the strength stabilization time, the time required to reach the peak strength can be more precisely predicted.

\section{Discussions}

Gypsum-cemented similar materials have been widely applied in mechanical simulation experiments in the mining field due to their advantages such as easy preparation and low cost. However, a standard protocol is still desired for such kind of experiment in the curing method. First, the curing duration is usually determined by personal experience [6]. The deviation introduced by empirical procedure undoubtedly results in errors in the estimation of material mechanical performance. Moreover, since regulations are missed in the curing procedure, significant differences can be identified from the variance in the curing temperature and humidity due to locations and seasons. Such variance introduced into the material strength may eventually affect the experimental results. Therefore, establishing a standard curing method and identifying the curing condition's influence on the material strength are highly desired by research community and industry.

The study indicates that the curing humidity has a significant influence on the similar material strength, which is mainly attributed to the fact that the humidity affects the water environment of gypsum hydration inside specimens. Thus, the humidity changes the supersaturation degree and the crystallization ability of gypsum and thereby affects the material compressive strength.

Introducing the curing model of the gypsum-cemented similar material into the analogous simulation experiment is beneficial to the standardization of curing method, which helps to improve the efficiency and accuracy of experiments. 


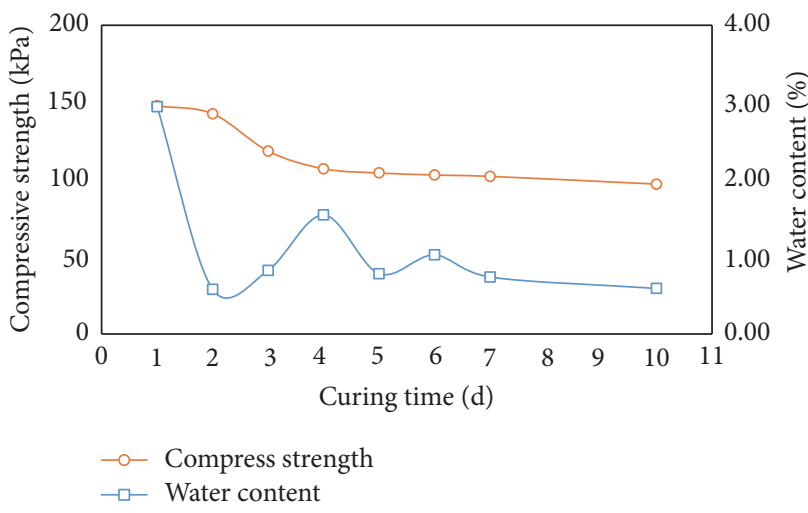

(a) $10 \%-15 \%$.

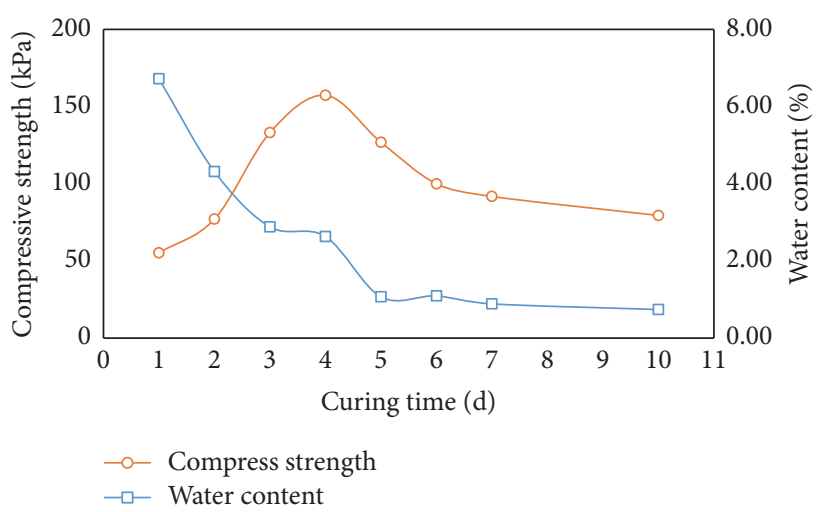

(c) $60 \%$.

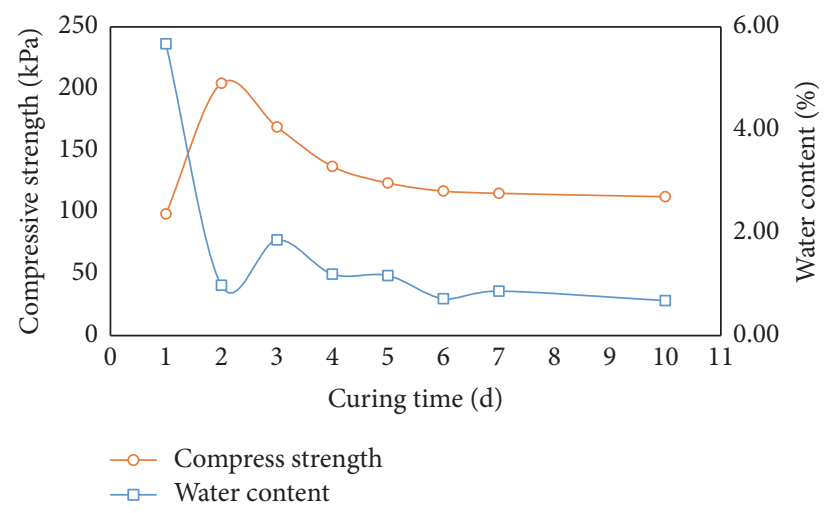

(b) $40 \%$.

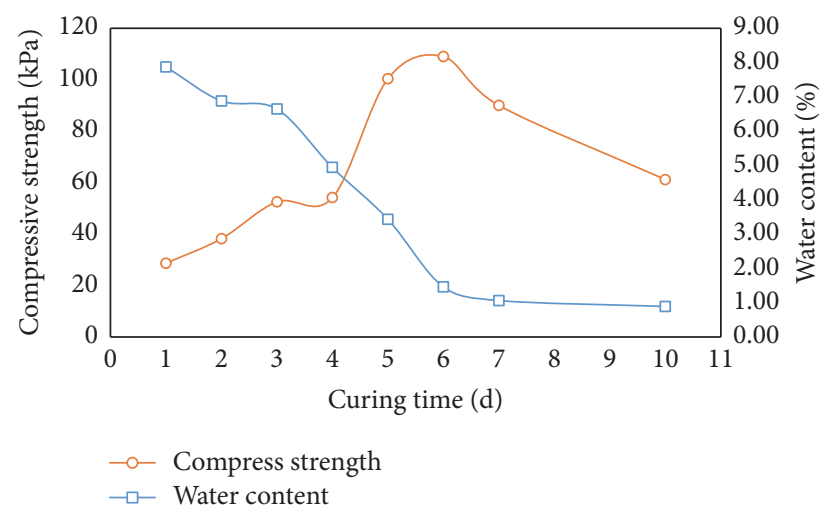

(d) $80 \%$.

FIGURE 12: Variation regularity of water content, strength, and time under various curing humidity.

Based on the curing method selection model, the most optimal curing humidity and shortest curing period could be selected to improve experiment efficiency. The material strength development stage will be predictable through continuous monitoring to material water content, which is also beneficial to the improvement of experiment efficiency.

In this study, soft rock and mud-rock are selected as the research subjects, which involve the deviations from different mixing ratios in the curing models of gypsum-cemented similar materials. Further study is required to clarify such deviation in future.

\section{Conclusions}

(1) The morphologies of hydration products inside gypsum-cemented similar materials change with curing duration. In the early stage of gypsum crystallization, needle-like phases are formed, which intersect in the late stage and form mat-like phases. The variation rate is affected by humidity; that is, the greater the humidity is, the more slowly the needle-like phase grows.

(2) During the hydration reaction of the gypsumcemented similar material, only semihydrate gypsum transforms into dihydrate gypsum. The other phases remain unchanged.
(3) The strength of the similar material first increases, then reduces, and finally becomes stable. The humidity has a significant influence on the variation rate, especially before peak strength is achieved. That is, the greater the humidity is, the lower the increase rate will be.

(4) The amplitudes and arrival time of peak material strength and stabilized average strength are influenced by the humidity. When the humidity is greater than $40 \%$, the strength decreases with reducing humidity. The opposite occurs when the humidity is lower than $40 \%$. The arrival time prolongs with increasing humidity. The transition humidity (40\%) differs with various mixing ratios, and further studies are required.

(5) Water content is an indirect index of the reaction degree of gypsum hydration. As time continues, the water content first rapidly reduces and then fluctuates to a stable value. The higher the humidity is, the lower the reduction rate will be. After the water content stabilizes, the material strength begins to reach its peak value. However, before the material strength reaches to the peak value, the water content is reduced and the strength is increased. Moreover, this change tendency prolongs with increasing humidity. After the strength reaches the peak value and begins to 
reduce, this change tendency follows the opposite trend.

(6) The influence of humidity on the strength variation of gypsum-cemented similar material is mainly attributable to the outward migration rate of water inside specimens. Thus, the humidity changes the supersaturation degree of gypsum. The internal crystallization state is changed, which finally reflects the variation of macroscopic strength.

(7) The curing methods in analogous simulation experiments should be regulated so as to establish curing sets to achieve control of the material, final strength, and arrival time. Thus, in further studies, the experimental processes can be standardized, and the test time can be reduced.

\section{Competing Interests}

The authors declare that there is no conflict of interests regarding the publication of this paper.

\section{Acknowledgments}

This study was supported by National Key Basic Research Program of China (973 Program, no. 2015CB251600) and National Natural Science Foundation of China (no. 51474206).

\section{References}

[1] X.-F. Wang, D.-S. Zhang, C.-G. Zhang, and G.-W. Fan, "Mechanism of mining-induced slope movement for gullies overlaying shallow coal seams," Journal of Mountain Science, vol. 10, no. 3, pp. 388-397, 2013.

[2] D. Zhang, G. Fan, and X. Wang, "Characteristics and stability of slope movement response to underground mining of shallow coal seams away from gullies," International Journal of Mining Science and Technology, vol. 22, no. 1, pp. 47-50, 2012.

[3] Q. Hu, S. Zhang, G. Wen, L. Dai, and B. Wang, "Coal-like material for coal and gas outburst simulation tests," International Journal of Rock Mechanics and Mining Sciences, vol. 74, pp. 151156, 2015.

[4] B. An, X. Miao, J. Zhang, F. Ju, and N. Zhou, "Overlying strata movement of recovering standing pillars with solid backfilling by physical simulation," International Journal of Mining Science and Technology, vol. 26, no. 2, pp. 301-307, 2016.

[5] M. Tao, X. Chen, and Q. Q. Ding, "A method for subsidence monitoring of similar material simulation test in coal mining," Advanced Materials Research, vol. 765-767, pp. 2172-2175, 2013.

[6] R.-S. Yang, Y.-F. Zhang, L.-Y. Yang, Y.-L. Wu, and J.-H. Ma, "Study on the mixing proportion test of similar material gypsum," China Mining Magazine, vol. 22, no. 10, pp. 125-130, 2013.

[7] X.-M. Shi, B.-G. Liu, and J. Xiao, "A method for determining the ratio of similar materials with cement and plaster as bonding agents," Rock and Soil Mechanics, vol. 36, no. 5, pp. 1357-1362, 2015.
[8] H. Wang, S. Li, Q. Zhang, Y. Li, and X. Guo, "Development of a new geomechanical similar material," Chinese Journal of Rock Mechanics and Engineering, vol. 25, no. 9, pp. 1842-1847, 2006.

[9] S. Li, X. Feng, S. Li, L. Li, and G. Li, "Research and development of a new similar material for solid-fluid coupling and its application," Chinese Journal of Rock Mechanics and Engineering, vol. 29, no. 2, pp. 281-288, 2010.

[10] S. Chen, H. Wang, J. Zhang, H. Xing, and H. Wang, "Experimental study on low-strength similar-material proportioning and properties for coal mining," Advances in Materials Science and Engineering, vol. 2015, Article ID 696501, 6 pages, 2015.

[11] X.-T. Kang, G. Huang, B.-Z. Deng, and P.-B. Han, "Experimental study on similar material for simulating raw coal," Journal of Northeastern University, vol. 36, no. 1, pp. 138-142, 2015.

[12] C. Ximin, M. Xiexing, S. Deguo, and M. Weimin, "Error analysis in similar material simulation test of the movement of rock strata and surface," Chinese Journal of Rock Mechanics and Engineering, vol. 21, no. 12, pp. 1827-1830, 2002.

[13] F. Luo, B.-S. Yang, B.-B. Hao, L.-H. Sun, and M.-M. Fu, "Mechanical properties of similar material under uniaxial compression and the strength error sources," Journal of Mining and Safety Engineering, vol. 30, no. 1, pp. 93-99, 2013.

[14] X. T. Zhang, Q. Y. Zhang, G. H. Cao, and X. B. Xu, "Effects on iron-barite-sand mixed similar material properties induced by molding pressure," Journal of Shandong University (Engineering Science), vol. 43, no. 2, pp. 89-95, 2013.

[15] B.-F. Li, Y.-K. Ren, L.-W. Qi, and L. Chang, "Study on orthogonal mixing rate experiment with low strength similar material of coal and rock mass," Coal Engineering, vol. 4, pp. 93-95, 2011.

[16] X. Jia, T. Li, H. Cao et al., "Influence of the sand particle size on the compressive strength in the analog model study," Journal of Soil Engineering and Foundation, vol. 1, pp. 83-86, 2016.

[17] P. Yuan and Y. Xu, "Influence of curing time on compressive properties of cemented sand similar materials," Journal of Vibration \& Shock, vol. 34, no. 13, pp. 200-204, 2015.

[18] L. I. Rui, "Climate characteristics analysis of relative humidity in urumqi in recent 40 years," Journal of Anhui Agricultural Sciences, vol. 27, pp. 15121-15122, 2010.

[19] Xuzhou Bureau of Statistics (NBS), Xuzhou Statistical Yearbook, China Statistics Press, Beijing, China, 2014.

[20] X. Li, Y. Lu, Y. Kang, and B. Rao, Rock Mechanics Test Simulation Technology, Science Press, Beijing, China, 2007. 

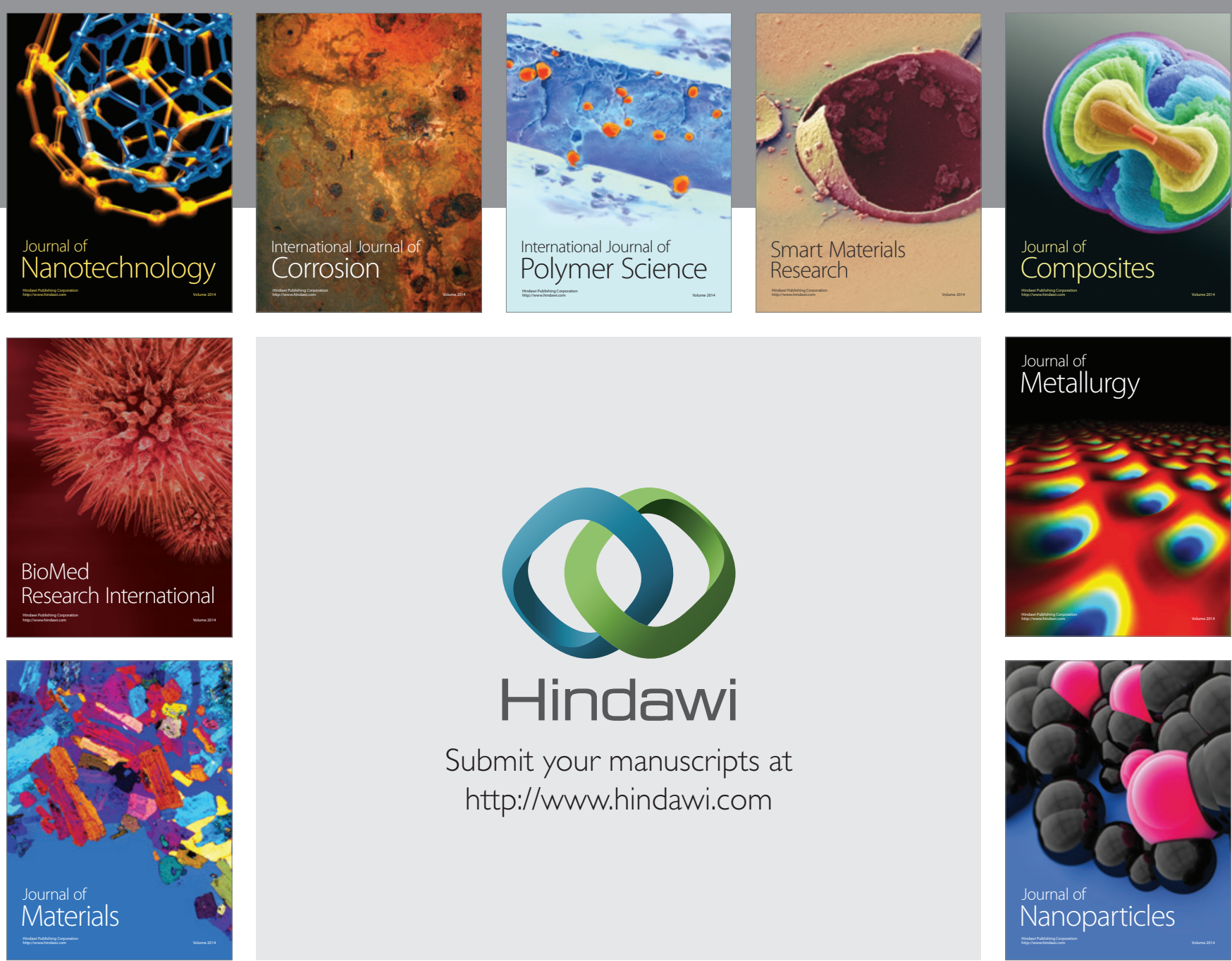

\section{Hindawi}

Submit your manuscripts at

http://www.hindawi.com

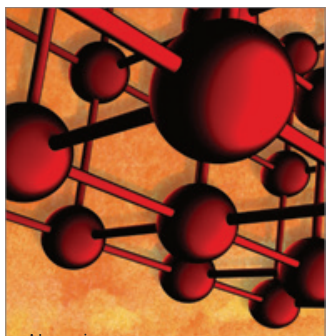

Materials Science and Engineering
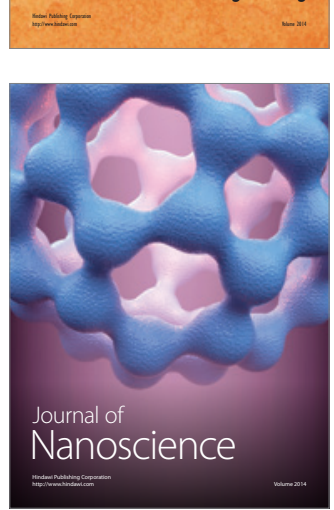
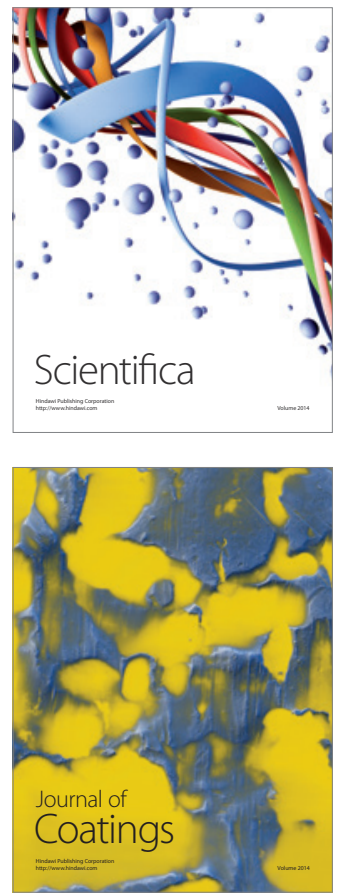
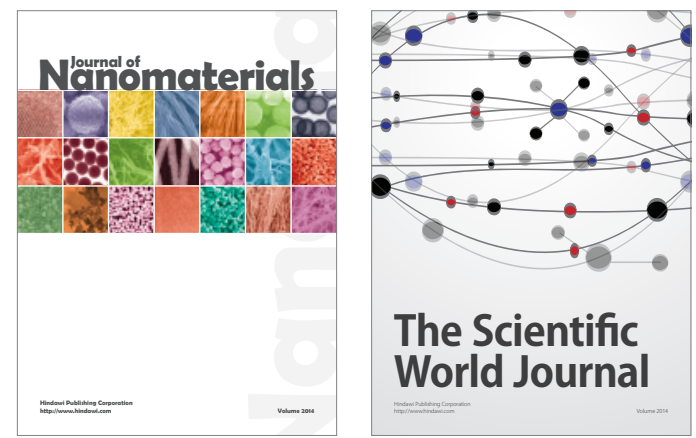

The Scientific World Journal
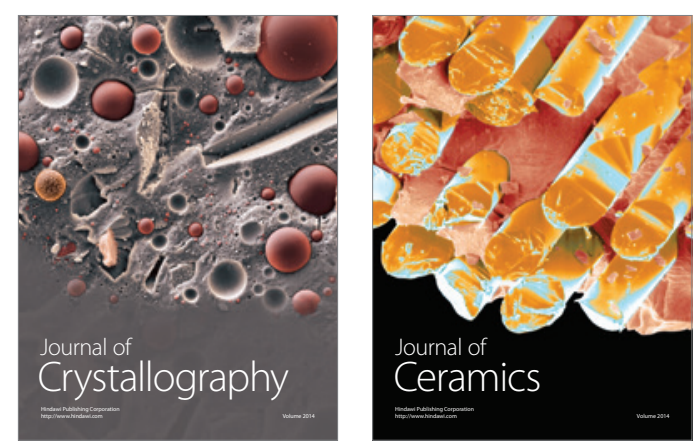
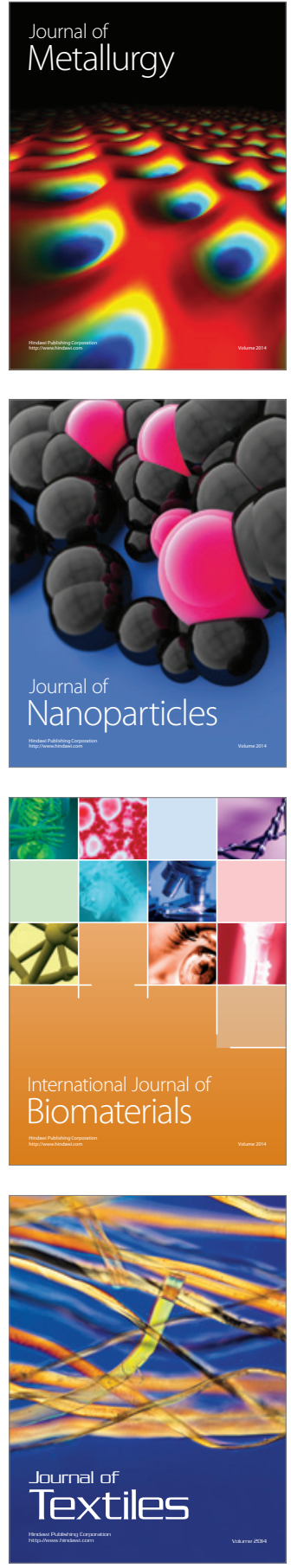